\title{
BMJ Open Caribbean Consortium for Research in Environmental and Occupational Health (CCREOH) Cohort Study: influences of complex environmental exposures on maternal and child health in Suriname
}

Wilco Zijlmans (1) , ,2,3 Jeffrey Wickliffe, ${ }^{3}$ Ashna Hindori-Mohangoo, ${ }^{3,4}$ Sigrid MacDonald-Ottevanger, ${ }^{2,5}$ Paul Ouboter, ${ }^{6}$ Gwendolyn Landburg, ${ }^{7}$ John Codrington, ${ }^{8}$ Jimmy Roosblad, ${ }^{1,8}$ Gaitree Baldewsingh, ${ }^{1,9}$ Radha Ramjatan, ${ }^{1,10}$ Anisma Gokoel, ${ }^{1,2}$ Firoz Abdoel Wahid, ${ }^{2,3}$ Lissa Fortes Soares, ${ }^{3}$ Cecilia Alcala, ${ }^{3}$ Esther Boedhoe, ${ }^{2}$ Antoon W Grünberg, ${ }^{4,11}$ William Hawkins, ${ }^{3,12}$ Arti Shankar, ${ }^{3}$ Emily Harville, ${ }^{13}$ S S Drury, ${ }^{14}$ Hannah Covert, ${ }^{3}$ Maureen Lichtveld ${ }^{3}$

To cite: Zijlmans W, Wickliffe J, Hindori-Mohangoo A, et al. Caribbean Consortium for Research in Environmental and Occupational Health (CCREOH) Cohort Study: influences of complex environmental exposures on maternal and child health in Suriname. BMJ Open 2020;10:e034702. doi:10.1136/ bmjopen-2019-034702

- Prepublication history for this paper is available online. To view these files, please visit the journal online (http://dx.doi. org/10.1136/bmjopen-2019034702).

Received 02 October 2019 Revised 28 July 2020

Accepted 04 August 2020

Check for updates

(C) Author(s) (or their employer(s)) 2020. Re-use permitted under CC BY-NC. No commercial re-use. See rights and permissions. Published by BMJ.

For numbered affiliations see end of article.

Correspondence to

Dr Wilco Zijlmans;

wilco.zijImans@uvs.edu

\section{ABSTRACT}

Purpose The Caribbean Consortium for Research in Environmental and Occupational Health prospective environmental epidemiologic cohort study addresses the impact of chemical and non-chemical environmental exposures on mother/child dyads in Suriname. The study determines associations between levels of environmental elements and toxicants in pregnant women, and birth outcomes and neurodevelopment in their children.

Participants Pregnant women $(\mathrm{N}=1143)$ were enrolled from December 2016 to July 2019 from three regions of Suriname: Paramaribo ( $N=738)$, Nickerie $(\mathrm{N}=204)$ and the tropical rainforest interior $(\mathrm{N}=201)$. Infants $(\mathrm{N}=992)$ were enrolled at birth. Follow-up will take place until children are 48 months old.

Findings to date Biospecimens and questionnaire data on physiological and psychosocial health in pregnant women have been analysed. $39.1 \%$ had hair mercury $(\mathrm{Hg})$ levels exceeding values considered safe by international standards. Median hair $\mathrm{Hg}$ concentrations in women from Paramaribo $(\mathrm{N}=522)$ were $0.64 \mu \mathrm{g} / \mathrm{g}$ hair (IQRs 0.36-1.09; range 0.00-7.12), from Nickerie $(\mathrm{N}=176) 0.73$ $\mu \mathrm{g} / \mathrm{g}$ (IQR 0.45-1.05; range 0.00-5.79) and the interior ( $\mathrm{N}=178) 3.48 \mu \mathrm{g} / \mathrm{g}$ (IQR 1.92-7.39; range 0.38-18.20). $96.1 \%$ of women ate fish, respective consumption of the three most consumed carnivorous species, Hoplias aimara, Serrasalmus rhombeus and Cichla ocellaris, known to have high $\mathrm{Hg}$ levels, was $44.4 \%, 19.3 \%$ and $26.3 \%$, respectively, and was greater among the interior subcohort. $89 \%$ frequently consumed the vegetable tannia, samples of which showed presence of worldwide banned pesticides. $24.9 \%$ of pregnant women had Edinburgh Depression Scale scores indicative of probable depression. Future plans Fish consumption advisories are in development, especially relevant to interior women for whom fish consumption is likely to be the primary source of $\mathrm{Hg}$ exposure. Effects of potentially beneficial neuroprotective factors in fish that may counter neurotoxic effects of $\mathrm{Hg}$ are being examined. A pesticide
Strengths and limitations of this study

- The study addresses two high-priority public health threats in Suriname and neighbouring countries in the Guiana Shield: the impact of mercury exposure from artisanal gold mining and pesticide exposure associated with agricultural practices in two vulnerable subpopulations: pregnant women and children younger than 5 years of age.

- The longitudinal follow-up of children to 48 months provides several timepoints to assess neurodevelopmental outcomes.

- The study has a biospecimen bank of approximately 13000 samples, providing the opportunity for future biomarker analyses.

- A linked research training grant facilitates the training of nine Surinamese $\mathrm{PhD}$ candidates with dissertation research embedded in the study, thereby building critical in-country environmental health research capacity.

- The interior subcohort $(\mathrm{N}=201)$ was logistically difficult to reach, resulting in delayed recruitment of some participants until the second or early third trimester. This may limit our ability to understand differential effects of exposure across gestation. Transportation challenges resulted in heel prick sampling replacing cord blood collection.

literacy assessment in pregnant women is in progress. Neurodevelopmental assessments and telomere length measurements of the children to evaluate long-term effects of prenatal exposures to toxicant mixtures are ongoing.

\section{INTRODUCTION}

The Caribbean Consortium for Research in Environmental and Occupational Health 
(CCREOH) addresses high-priority environmental and occupational health risks in Suriname and those common to the vulnerable Caribbean region, while preserving unique cultural traditions of indigenous people and other health disparate populations. ${ }^{1}$ Exposure to environmental contaminants at levels of public health concern may adversely affect pregnancy, and prenatal and postnatal health in multiple ways: miscarriage, preterm delivery, intrauterine growth retardation, congenital anomalies and behavioural and physical consequences in later developmental stages. ${ }^{2-4}$ Prenatal exposure to multiple heavy metals is associated with adverse paediatric health outcomes. ${ }^{5}$ Pesticide exposure has been linked to fetal growth decrements and preterm birth, and there is mounting evidence that exposure to pesticides during prenatal and postnatal development is associated with neurodevelopmental deficits in young children ${ }^{6-10}$ Exposure to contaminant mixtures can exacerbate adverse health effects: for example, lead, arsenic and mercury $(\mathrm{Hg})$ can potentiate each other's toxicity, even at individual levels below concentration expected to result in adverse effects. ${ }^{11}$ Despite this knowledge, no cumulative risk assessments have been conducted to date addressing exposures to contaminant mixtures in Caribbean low-income and middle-income countries. ${ }^{12}$

The CCREOH environmental epidemiology cohort study fills this gap by exploring the impact of exposures to organic and inorganic neurotoxicants, including $\mathrm{Hg}$, lead and multiple organophosphate pesticides, on Surinamese pregnant women and their offspring using a cumulative risk approach. Specifically, the study assesses the impact of exposures to chemical and non-chemical stressors by examining the interaction between exposure to environmental chemicals and social and psychological determinants of early neurodevelopment. Few prospective studies have measured exposure prenatally through 48 months in mother/child dyads, and those that have showed contrasting results when evaluating executive function, a key neurodevelopmental outcome. ${ }^{13-16}$ Our hypothesis is that environmental exposures to mixtures of neurodevelopmental toxicants and non-chemical stressors will result in increased adverse birth outcomes and poorer child neurodevelopmental trajectories. Our research aims are to:

- Identify exposures to a complex mixture of environmental elements and toxicants, including $\mathrm{Hg}$, lead, cadmium, aluminium, iron, manganese, tin, selenium and selected pesticides through comprehensive dietary and environmental risk assessments, and biomarker monitoring in Surinamese pregnant women and their offspring.

- Assess levels of environmental elements and toxicants in fish, produce, rice and nutraceutical compounds.

- Determine the association between levels of environmental elements and toxicants in pregnant women and birth outcomes.

- Assess the impact of mobile health technologyenabled community health workers (CHWs) on birth outcomes and their associations with environmental contaminants.

- Determine associations between prenatal, dietary and environmental levels of elements and toxicants and potential neuroprotective nutraceuticals on neurodevelopment.

CCREOH is funded by the Fogarty International Center at the US National Institutes of Health (NIH).

\section{COHORT DESCRIPTION}

\section{Study area}

The Republic of Suriname is located on the northeastern coast of South America, bordered by Brazil, Guyana, French Guiana and the Atlantic Ocean. Overall, 90\% of the 590549 population lives in the capital Paramaribo and the coastal area. The remainder lives in the tropical rainforest interior (90\% of the landmass). Suriname's multiethnic population consists of five main groups: Hindustani (27\%), Tribal (22\%), Creole (16\%), Javanese $(14 \%)$ and Indigenous $(4 \%) .{ }^{17}$

\section{Recruitment}

Pregnant women potentially exposed to complex environmental chemical and non-chemical stressors were recruited from three regions of Suriname: (1) Paramaribo, where pesticides are primarily used for residential purposes; (2) Nickerie, the major rice producing region in western Suriname where pesticide use is abundant and (3) the tropical rainforest interior, where $\mathrm{Hg}$ is used in artisanal gold mining and the population is highly dependent on consumption of contaminated fish. ${ }^{18}$ Taking into account, a potential $20 \%$ lost to follow-up, we requested and were approved to consent 1200 pregnant women and their singleton birth children by the Institutional Review Boards of both the Government of Suriname and Tulane University.

Eighty-five per cent of all deliveries in Suriname are hospital based, with the remainder (14\%) taking place in primary healthcare clinics under the supervision of a general practitioner or midwife at the Regional Health Department or a skilled healthcare worker at the Medical Mission Primary Health Care Suriname; a small proportion $(1 \%)$ occurs at home. Recruitment sites included all hospitals and clinics of the Regional Health Department in Paramaribo and Nickerie and the Medical Mission Primary Health Care Suriname in the interior. In Nickerie, trained CHWs were integrated in the research team and played a key role in every aspect of the study, from recruitment to assessments at every study timepoint prenatally and during the neurodevelopmental assessments of the child cohort. Women who met the inclusion criteria were identified by their physician, midwife, CHW or health assistant during regular prenatal appointments and invited to participate. Inclusion criteria were: pregnant women 16 years or older; speaking Dutch, Sranan Tongo, Sarnami, Saramaccan or Trio; singleton gestation; planning to deliver at one of the study hospitals, 
prenatal clinics or midwife facilities associated with those hospitals or clinics; and signed informed consent. Assent was obtained from participants aged 16 and 17 years. Informed consent forms and questionnaires were translated into the local languages Sranan Tongo, Sarnami, Saramaccan or Trio. If a participant was unable to read, the recruiter read the questions in the local language.

Infants were not eligible for follow-up if they were born before 33 completed weeks of gestation and/or had a birth weight less than $2000 \mathrm{~g}$, significant medical or neurological condition, down syndrome, hydrocephalus, cerebral palsy or significant visual or hearing impairment inconsistent with neurocognitive testing.

Main maternal and infant/child determinants included biological determinants: maternal anthropometrics, blood pressure, haemoglobin, liver and kidney function, fetal and postnatal growth characteristics and health status, heavy metals (blood and hair), pesticides (urine), telomere length (TL) (buccal swab) and medication use; environmental determinants: maternal and child diet and exposure history; social determinants: ethnicity, social support, trauma, prenatal life events, maternal education, household income, household size, employment status and marital status. Main maternal and infant/child outcomes were growth and physical development: pregnancy complications, fetal and postnatal growth patterns, risk factors for maternal liver and renal impairment; behaviour and cognitive development: infant neuromotor development, autism spectrum disorder and neuropsychological development; childhood diseases: infectious diseases, respiratory and neurological disorders; health and healthcare: impact of mobile health technologyenabled CHWs on prenatal health and birth outcomes, quality-of-life assessments and the impact of healthcare system utilisation on prenatal health and birth outcomes.

\section{Data management and statistical plan}

The CCREOH field team was trained in administering all questionnaires. Data from administered questionnaires are recorded on secure, encrypted iPads, uploaded and managed using Research Electronic Data capture (REDCap), which serves as the study electronic, integrated data management system. ${ }^{19}$ Data are uploaded in REDCap's training site for data cleaning prior to integrating questionnaire and biospecimen data. Data records are maintained to examine trends on source of errors, duplicate records are scrubbed, and accuracy is validated through crosschecks with original data files and medical records obtained through the general practitioner, midwife, health assistant or hospital administration. A comprehensive biospecimen tracking system tracks analysed samples and the study's overall biospecimen repository. Communication of data updates and changes in the process are delivered through emails and during bimonthly data management team meetings. The data management platform allows for the interrogation of environmental, non-biospecimen and biospecimen data in an integrated fashion.
Deidentified data files are developed on request of CCREOH investigators through standardised data request forms. Statistical analysis plans are tailored to specific research questions and include descriptive statistics, bivariate linear and logistic regression models, multiple logistic regression analysis to develop predictive models, and non-parametric methods if assumptions are not met. Comparison between study sites will be analysed by comparing participants, or clustered depending the research question.

Multiple approaches to develop and compare models for multiple exposures to toxic metals will be used including a Bayesian kernel machine regression and a non-parametric Bayesian variable selection framework to explore joint (or combined) effects of the multiple chemicals. Structural equation models will be employed to create latent constructs of similar exposure variables and then finding associations between these latent constructs and the outcome variable. Sample size was calculated based on a multiple linear regression model using a coefficient of determination of 0.10 and an $\mathrm{R}^{2}$ differential ranging between 0.01 and 0.02 . Using these parameters, a number between 495 and 986 was needed to have an $80 \%$ power at a 0.05 level of significance. Using the more conservative $\mathrm{R}^{2}$ differential of 0.02 and to account for attrition we planned on recruiting around 1200 participants.

\section{Data collection}

Maternal data were collected during pregnancy at two timepoints (first/second and third trimester). Postpartum assessments are ongoing and target both mothers and children: questionnaire data from mothers and biospecimens and neurodevelopmental assessments data from children are collected at 12, 36 and 48 months (table 1).

\section{Data collection during pregnancy \\ Questionnaires}

Table 1 lists standardised validated questionnaires used to assess both physiological and psychosocial prenatal healths. All questionnaires were translated into Dutch and other local languages to address the multiple language needs of our interior subcohort. The main categories of data collected from maternal participants were health status, demographics, reproductive health history, social support, trauma history, exposure history, depression, perceived stress, prenatal life events, health behaviour, access to prenatal care, social status and diet. Questions on health behaviour were adapted from the Alcohol, Smoking and Substance Involvement Screening Test version V.3 that was developed for WHO. Information on maternal diet was obtained by adapting the Centers for Disease Control and Prevention (CDC)'s National Health and Nutrition Examination Survey (NHANES) into a culturally tailored dietary survey focusing on fish and produce consumption, including frequency of intake and portion sizes. 
Table 1 Assessments completed by the CCREOH-MeKi Tamara Cohort with timeline

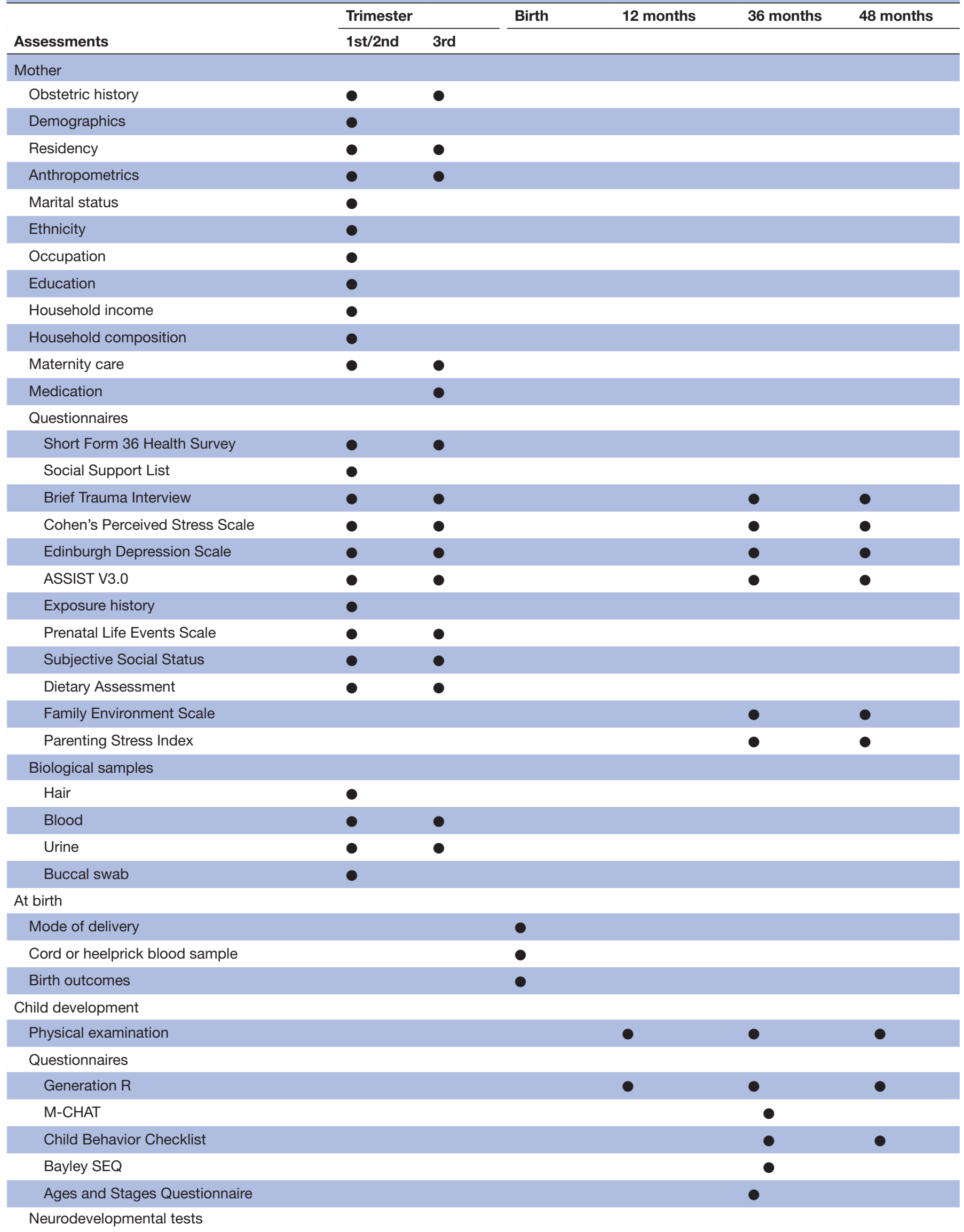


Table 1 Continued

\begin{tabular}{|c|c|c|c|c|c|c|}
\hline \multirow[b]{2}{*}{ Assessments } & \multicolumn{2}{|c|}{ Trimester } & \multirow[t]{2}{*}{ Birth } & \multirow[t]{2}{*}{12 months } & \multirow[t]{2}{*}{36 months } & \multirow[t]{2}{*}{48 months } \\
\hline & 1st/2nd & 3rd & & & & \\
\hline BSID-III & & & & $\bullet$ & & \\
\hline \multicolumn{7}{|c|}{ Biological samples } \\
\hline Buccal swab & & & & $\bullet$ & 0 & 0 \\
\hline Urine & & & & & - & - \\
\hline
\end{tabular}

ASSIST V3.0, Alcohol, Smoking and Substance Involvement Screening Test Version 3; Bayley SEQ, Bayley Social Emotional Questionnaire; BSID-III, Bayley Scales of Infant Development third edition; CANTAB, Cambridge Neuropsychological Test Automated Battery; Generation R, questionnaires on paediatric health and diet history (with permission from The Generation R Study Group); M-CHAT, Modified Checklist for Autism in Toddlers; Subjective Social Status, MacArthur Scale of Subjective Social Status.

\section{Biospecimen collection}

The study's biospecimen repository, housed at the Academic Hospital Paramaribo's (AZP's) clinical laboratory, contains 13000 samples. Table 1 lists timepoints at which biospecimens are collected. All biospecimens are collected at study site hospitals and shipped to the AZP laboratory using established chain-of-custody procedures. Samples collected from participants in remote areas are stored at $4^{\circ} \mathrm{C}$ for not more than 48 hours prior to delivery and processing at the AZP clinical laboratory. Blood, serum, plasma and cord blood samples are aliquoted into $2 \mathrm{~mL}$ plastic freezer tubes and stored at $-80^{\circ} \mathrm{C}$. Urine samples $(60 \mathrm{~mL})$ are collected in sterile bar-coded urine collection cups, aliquoted into $10 \mathrm{~mL}$ plastic tubes, stored in ziplock bags at $-20^{\circ} \mathrm{C}$ and shipped for analysis by CDC's environmental health laboratory using well-established protocols.

Maternal hair samples, collected once during pregnancy, were taken according to the following protocol: $1.5 \mathrm{~g}$ of hair was cut as close as possible to the hair roots with disinfected scissors and placed in patient-coded ziplock bags, stored at room temperature in a climate-controlled room and sent to the National Zoological Collection of Suriname/Center for Environmental Research lab at the Anton de Kom University of Suriname for total $\mathrm{Hg}$ analysis using Cold Vapour Atomic Absorption Spectrometry.

\section{Buccal swab collection for telomere assessments}

Isohelix SK1 buccal swabs (Cell Projects, Kent, United Kingdom) are used for the collection of maternal DNA for TL analyses. Swabs are air dried, and then stored with a dessicator pellet at $4^{\circ} \mathrm{C}$ until DNA is extracted. DNA is extracted using the QIAamp DNA mini kit protocol (Qiagen, Valencia, CA, USA) and stored at $-80^{\circ} \mathrm{C}$ until assayed. Concentration of dsDNA is quantified with a Qubit dsDNA Broad Range (BR) assay kit (Invitrogen, Carlsbad, CA, USA), purity of the DNA is determined by using a NanoDrop 1000 spectrophotometer (Thermo Fisher Scientific, Waltham, MA, USA) and DNA integrity is confirmed by gel electrophoresis to ensure high molecular weight DNA. ${ }^{20}$ The average relative TL, represented by the telomere repeat copy number to single gene (albumin) copy number $(\mathrm{T} / \mathrm{S})$ ratio, is determined with monochrome multiplex quantitative real-time PCR using a BioRad CFX V.96. ${ }^{21}$ All samples are run in triplicate on duplicate plates with a standard curve and known controls.

\section{Trace element analyses in blood}

Four hundred frozen maternal whole blood samples were shipped for analysis to the Wisconsin State Laboratory of Hygiene Trace Element Research Laboratory. A chainof-custody approach was used to ensure samples were collected, maintained, processed, stored, shipped and received according to acceptable standards. Quality assurance/quality control (QA/QC) procedures for elemental analyses and methylmercury analyses included reagent blanks, blank spikes (lab fortified blanks), sample matrix spikes, ongoing precision and recovery spikes, second source spikes, sample matrix duplicates and external standard reference materials. All sample and QA/QC results were within the acceptable recovery limits. Duplicates for all runs were within the acceptable relative percent difference limits. In these 400 blood samples (200 Paramaribo, 100 Nickerie and 100 interior communities), we analysed $\mathrm{Hg}$, lead, cadmium, aluminium, manganese, tin, selenium and iron using inductively coupled plasma-mass spectrometry or cold vapour atomic fluorescence spectrometry (CVAFS). Total $\mathrm{Hg}$ and methylmercury using CVAFS were assessed in a small subset of women (20Paramaribo, 20-Nickerie and 35-interior) to specify exposure sources.

\section{Pesticide analyses in urine}

Urine samples from pregnant women were analysed (subcohort, $\mathrm{N}=218$ ) from all three study sites. The six dialkylphosphate metabolites were measured using a modified method of Jayatilaka $e t a t^{22}$ by solid-phase extraction high-performance liquid chromatographytandem mass spectrometry. The eight specific urinary metabolites were analysed using a semiautomated solid-phase extraction mass spectrometry method and 
reversed-phase high-performance liquid chromatography. ${ }^{23}$ The analysis panel consisted of dialkylphosphate metabolites of organophosphate pesticides including dimethylphosphate, diethylphosphate, dimethylthiophosphate, dimethyldithiophosphate, diethylthiophosphate and diethyldithiophosphate, and a universal panel including one herbicide (2,4D) and its metabolite, 2,4-dichlorophenoxyacetic acid; four organophosphates (OP) insecticide metabolites (3,5,6-trichloro-2-pyridinol, 2-isopropyl-4-methyl-6-hydroxypyrimidine, paranitrophenol and malathion dicarboxylic acid) and three pyrethroid metabolites (4-fluoro-3-phenoxybenzoic acid, 3-phenoxybenzoic acid and trans-3-(2,2-dichlorovinyl)2,2-dimethylcyclopropane carboxylic acid).

\section{Data collection from birth through $\mathbf{4 8}$ months}

At birth, a cord blood sample was taken from infants for measurements of heavy metals. When a cord blood sample was not available, a blood sample was obtained by heel prick. Baseline characteristics of all births (eg, gender, weight, gestational age and Apgar Score) were collected (table 2). At each subsequent data collection timepoint (table 1), the child's growth is measured and a buccal swab taken for TL analyses. In addition, the child's health status is obtained through questionnaires on diet, history of infectious diseases and respiratory and neurological disorders. To date, we have completed the first follow-up with $85 \%$ of children. A blood sample will be taken for heavy metal measurement at 36 and 48 months. Blood samples and buccal swabs are collected and stored in the same manner as described above for mothers.

\section{Behaviour and cognitive development}

Neurodevelopmental assessments over the lifetime of the study include infant cognitive and motor development and behaviour at 12-27 months using the Bayley Scales of Infant Development as well as assessments of cognitive and social-emotional development at 36 months, and executive function at 48 months (table 1). The Bayley Scales are currently being administered to enrolled children by trained psychologists, psychiatrists and study team members. Data checking following data entry of each Bayley subscale is conducted by other team members. To date, 832 of 992 children have been tested; remaining assessments will be completed by September 2020. Assessments at 36 and 48 months will take place as the cohort ages.

\section{Data collection from fish}

Carnivorous fish from different regions in the interior of Suriname have shown high levels of $\mathrm{Hg}$, specifically the frequently consumed species Hoplias aimara (0.43$0.66 \mu \mathrm{g} / \mathrm{g})$ and Serrasalmus rhombeus $(0.23-1.38 \mu \mathrm{g} / \mathrm{g})$. These levels are well above international accepted standards and in certain regions, up to seven times the norm for human consumption. ${ }^{24}$ Polyunsaturated omega-3 fatty acids, docosahexaenoic acid (DHA) and eicosapentaenoic acid (EPA) from fish consumption may offer neuroprotective benefits during prenatal and paediatric development which could potentially counteract $\mathrm{Hg}$ neurotoxicity. ${ }^{25-27}$ Some studies examining developmental trajectories of children born to women exposed to $\mathrm{Hg}$ in utero fail to link that exposure to adverse neurodevelopmental outcomes, suggesting that fish nutrients may ameliorate $\mathrm{Hg}$ toxicity and offer neuroprotective benefits. $^{28-31}$

To better inform fish consumption risk, fatty acids in fish muscle tissue were measured in samples $(\mathrm{N}=5$ per species) of five freshwater and three marine fish species. The levels of 27 fatty acids, including DHA and EPA, in fish muscle tissue were measured (expressed as $\mathrm{mg}$ fatty acid per gram dry weight) by the University of Texas Marine Science Institute. Fifty milligrams dry weight samples were homogenised in a chloroform-methanol solution $(2: 1 \mathrm{v} / \mathrm{v})$ for lipid cold extraction. Lipids were then saponified in potassium hydroxide to yield fatty acid methyl esters, and then methylated in a $14 \%$ boron trifluoride solution. Individual fatty acid concentrations were determined by gas chromatography (Shimadzu GC-2014 gas chromatograph) set with a Supelcowax V.10 fused silica capillary column (Milipore Sigma). Freshwater species had higher levels of linoleic acid ( $2.0 \mathrm{vs} 0.2 \mathrm{mg} / \mathrm{g}$ ), alpha-linoleic acid ( 0.4 vs $0.1 \mathrm{mg} / \mathrm{g}$ ), arachidonic acid (3.0 vs $1.5 \mathrm{mg} / \mathrm{g}$ ), omega-6 fatty acids (6.5 vs $2.3 \mathrm{mg} / \mathrm{g}$ ) and lower levels of EPA ( $0.8 \mathrm{vs} 1.9 \mathrm{mg} / \mathrm{g}$ ) compared with marine species.

\section{PATIENT AND PUBLIC INVOLVEMENT}

The CCREOH Cohort Study was preceded by a planning grant which included pilot fish and hair $\mathrm{Hg}$ assessments among interior communities approved by the village Chiefs or Tribal captains. Building on this community partnership, the initial study design was revised based on guidance from a multistakeholder Community Advisory Board $(\mathrm{CAB})$ and an External Scientific Advisory Board (EAB). The $\mathrm{CAB}$ consists of respected leaders representing organisations from the interior tribal and indigenous peoples, midwives, general practitioners and environmentalists. The EAB members represent medical directors and other clinicians of all participating hospitals, representatives from the Ministry of Health, the US Embassy, the Pan American Health Organization and the Caribbean Public Health Agency (CARPHA). Both advisory boards are convened annually to inform and give updates about study progress and results, and to seek advice on future directions and dissemination of results.

\section{Dissemination of study results}

Every participant with neurotoxicant levels of imminent health concern is contacted and asked to repeat the test for confirmation. If the level remains of concern, the participant is referred to her attending physician. Children with neurodevelopmental delay are referred to a child psychologist. Participants and the general public were invited to health fairs, meetings and conferences, 
Table 2 Distribution of maternal and infant characteristics

\begin{tabular}{|c|c|c|c|c|c|}
\hline Maternal characteristics $(n=1143)$ & $\mathbf{N}$ & $\%$ & Infant characteristics $(n=971)$ & $\mathbf{N}$ & $\%$ \\
\hline Age at intake & & & Gender & & \\
\hline 16-19years & 142 & 12.6 & Male & 510 & 52.5 \\
\hline 20-24 years & 260 & 22.8 & Female & 457 & 47.1 \\
\hline 25-29years & 291 & 25.5 & Missing & 4 & 0.4 \\
\hline 30-34 years & 268 & 23.5 & Birth status & & \\
\hline 35-39years & 140 & 12.3 & Live birth & 947 & 97.5 \\
\hline $40+$ years & 39 & 3.4 & Stillbirth & 23 & 2.4 \\
\hline Missing & 1 & 0.1 & Missing & 1 & 0.1 \\
\hline Parity & & & Birth weight (in grams) & & \\
\hline 0 previous live births & 384 & 33.7 & Low birth weight $(<2500)$ & 127 & 13.1 \\
\hline One previous live birth & 312 & 27.3 & Normal birth weight $(\geq 2500)$ & 835 & 86.0 \\
\hline Two previous live births & 187 & 16.4 & Missing & 9 & 0.9 \\
\hline Three previous live births & 112 & 9.8 & Gestational age (in weeks) & & \\
\hline 4+previous live births & 146 & 12.8 & Very preterm births $(22+0-32+6)$ & 39 & 4.0 \\
\hline \multirow[t]{2}{*}{ Missing } & 2 & 0.2 & Moderately preterm births $(33+0-36+6)$ & 107 & 11.0 \\
\hline & & & Term births $(\geq 37+0)$ & 817 & 84.1 \\
\hline Ethnicity & & & Missing & 8 & 0.8 \\
\hline Creole & 249 & 21.8 & Apgar score at $5 \mathrm{~min}$ & & \\
\hline Hindustani & 233 & 20.4 & $0-6$ & 31 & 3.2 \\
\hline Indigenous & 155 & 13.6 & $7-10$ & 918 & 94.5 \\
\hline Javanese & 101 & 8.8 & Missing & 22 & 2.3 \\
\hline Tribal & 271 & 23.7 & & & \\
\hline Mixed & 127 & 11.1 & & & \\
\hline Other & 7 & 0.6 & & & \\
\hline Missing & 0 & 0.0 & & & \\
\hline \multicolumn{6}{|l|}{ Educational level } \\
\hline No or primary & 276 & 24.1 & & & \\
\hline Lower vocational/secondary & 382 & 33.4 & & & \\
\hline Upper vocational/secondary & 317 & 27.7 & & & \\
\hline Tertiary & 168 & 14.7 & & & \\
\hline Missing & 0 & 0.0 & & & \\
\hline \multicolumn{6}{|l|}{ Household income SRD* } \\
\hline$<1500$ & 401 & 35.1 & & & \\
\hline 1500-2999 & 362 & 33.0 & & & \\
\hline $3000-4999$ & 221 & 20.2 & & & \\
\hline $5000+$ & 112 & 9.8 & & & \\
\hline Missing & 47 & 4.1 & & & \\
\hline \multicolumn{6}{|l|}{ Marital status } \\
\hline Married or living with partner & 1000 & 87.6 & & & \\
\hline Not married/not living with partner & 141 & 12.4 & & & \\
\hline Missing & 2 & 0.2 & & & \\
\hline
\end{tabular}

*Surinamese Dollar, equivalent to US\$0.13.

SRD, Surinamese Dollar.

where preliminary study results were presented and discussed. CCREOH data have been presented at regional and international conferences, including CARPHA, the
International Society of Environmental Epidemiology, the Consortium for Universities in Global Health, the American Public Health Association and at scientific meetings 


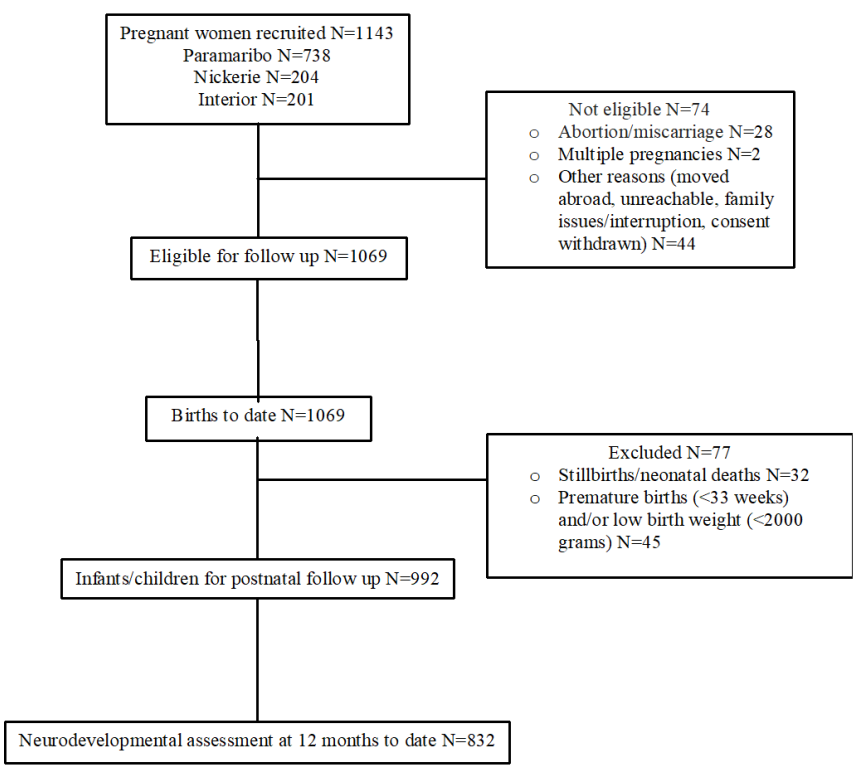

Figure 1 Flowchart with participant enrolment.

convened by the US NIH. The study team is working with the $\mathrm{EAB}$ and $\mathrm{CAB}$ to develop a collaborative translation and dissemination strategy for overall, final study results when those become available.

\section{FINDINGS TO DATE}

From December 2016 until July 2019, 1143 pregnant women were enrolled; $74(6.5 \%)$ were ineligible (figure 1). Geographically, 738 women were enrolled in Paramaribo, 204 in Nickerie and 201 in the interior. In comparison with participants, non-participants were more often of Creole and Tribal ethnicity, lived in Paramaribo and had lower household income. Table 2 presents baseline maternal characteristics: one in eight women was 16-19 years old, ethnic distribution was representative of the Surinamese population, and $>50 \%$ had no or lower vocational/secondary education level.

Of the 1069 babies born, 992 were enrolled (93\%) (figure 1). To date, the total number of biospecimens $(\mathrm{N}=13379)$ collected from 1143 pregnant women includes whole blood for trace elements $(\mathrm{N}=1994)$, whole blood collected in dipotassiumethylenediaminetetraacetic acid (K2EDTA) anticoagulant $(\mathrm{N}=1994)$, serum collected in serum separator tubes $(\mathrm{N}=1994)$, plasma $(\mathrm{N}=1994)$, urine $(\mathrm{N}=1980)$, buccal swabs $(\mathrm{N}=941)$ and hair $(\mathrm{N}=876)$. From 992 infants, either cord blood $(\mathrm{N}=441)$ or blood from heel prick $(\mathrm{N}=323)$ was collected at birth, and buccal swabs ( $\mathrm{N}=842)$ during neurodevelopmental assessment at 12 months. All samples not yet analysed are archived for future targeted and untargeted analyses. Available results include total $\mathrm{Hg}$ concentrations in hair, prenatal maternal depression and perceived stress, level of pesticides in produce, and dietary exposure to $\mathrm{Hg}$ in fish. The results of heavy metals in blood, urinary pesticide metabolites, telomere analysis and neurodevelopmental assessments are pending.

\section{Biological specimens}

Total hair $\mathrm{Hg}$ was measured in 876 participants from all 3 study sites. Overall, $39.1 \%$ had elevated total $\mathrm{Hg}$ hair levels that exceeded the United States Environmental Protection Agency's action level $(1.1 \mu \mathrm{g} / \mathrm{g}$ hair $) .{ }^{32}$ Elevated $\mathrm{Hg}$ levels were found in $95.8 \%, 26.3 \%$ and $25.5 \%$ of participants from the interior, Nickerie and Paramaribo, respectively. The results for median total $\mathrm{Hg}$ concentrations in hair from pregnant women from Paramaribo $(\mathrm{N}=522)$ were $0.64 \mu \mathrm{g} / \mathrm{g}$ hair (IQRs $0.36-1.09$; range $0.00-7.12$ ), from Nickerie $(\mathrm{N}=176) 0.73 \mu \mathrm{g} / \mathrm{g}$ (IQR $0.45-1.05$; range $0.00-5.79$ ) and the interior $(\mathrm{N}=178) 3.48 \mu \mathrm{g} / \mathrm{g}$ (IQR 1.92-7.39; range 0.38-18.20). Pregnant women from the interior were exposed to high levels of $\mathrm{Hg}$ compared with women from the coastal area, and thus fetal exposures are expected to be high. Most of these women are primarily exposed to methylmercury from consuming contaminated fish from local artisanal gold-mining activities. ${ }^{182}$ Other possible sources of exposure may include active participation in or living very close to gold mining areas. $\mathrm{Hg}$ concentrations from the Nickerie and Paramaribo subcohorts were similar to other studies. ${ }^{33} 34$

\section{Prenatal depression and perceived stress}

Depression and prenatal stress in 722 participants were assessed using the standardised Edinburgh Depression Scale (EDS) (cut-off $\geq 12$ for probable depression) and the Cohen Perceived Stress Scale (cut-off $\geq 20$ for high perceived stress). One in four $(24.9 \%)$ participants had EDS scores indicative of probable depression, three in ten $(30.2 \%)$ had high stress levels and nearly half $(49.1 \%)$ of these women had probable depression.

\section{Dietary exposure to pesticides}

An assessment of Surinamese agricultural produce showed pesticide residues exceeding European Union maximum residue limits, including prohibited worldwide endosulfan and lindane in the leafy vegetable tannia, Xanthosoma brasiliense. ${ }^{35}$ An interviewer-assisted NHANESbased dietary survey of 522 participants showed that $98.2 \%$ reported consumption of leafy vegetables. Tannia was the most frequently consumed $(89.3 \%) ; 36.5 \%$ participants had high intake rates of tannia ( $\geq 36 \mathrm{~g}$ /day) ${ }^{36}$ Tannia is also a commonly used vegetable in baby food preparation in Suriname.

\section{Dietary exposure to $\mathrm{Hg}$ in fish}

Dietary questionnaire analyses $(\mathrm{N}=990)$ showed an overall fish consumption of $96.1 \%$. Consumption of the three most consumed carnivorous species-H. aimara, S. rhombeus and Cichla ocellaris-, known to have high $\mathrm{Hg}$ levels, was $44.4 \%, 19.3 \%$ and $26.3 \%$, and was greater among the interior subcohort $(\mathrm{N}=123): 89.4 \%, 67.5 \%$ and $74.8 \%$, respectively. Intake rates (based on reported meal frequency and portion sizes) for these three carnivorous species ranged between 0.01 and $2.5 \mathrm{~kg}$ /week. $\mathrm{Hg}$ 
speciation indicated that methylmercury was predominantly found in the biospecimens. This is consistent with fish consumption likely being the primary source of $\mathrm{Hg}$ exposure in our participants.

\section{Strengths and limitations}

This CCREOH study is the first to examine the potential impact of complex environmental exposures on maternal and child health in Suriname by a multidisciplinary team of scientists. Study's strengths include: (1) addressing two high-priority public health threats: the impact of $\mathrm{Hg}$ exposure from artisanal gold mining, and pesticide exposure associated with agricultural practices; (2) inclusion in the cohort of two vulnerable subpopulations: pregnant women and children; (3) the longitudinal follow-up of children until 48 months with built-in timepoints to assess neurodevelopmental outcomes and (4) a biospecimen bank of approximately 13000 samples, providing the opportunity for future analyses. Moreover, a linked research training grant facilitates the training of nine Surinamese $\mathrm{PhD}$ candidates with dissertation research embedded in the study, thereby building critical in-country environmental health research capacity.

Delayed recruitment and data collection of the logistically difficult to reach subcohort in the interior region $(\mathrm{N}=200)$ were ameliorated by additional team support onsite; delays in transporting interior biospecimens rendered some early cord blood specimens not analysable and was corrected by collecting an additional heelprick sample. Other limitations include differences in educational level between women from the three recruitment sites that could confound or impact literacy. Participants may have moved between regions with different sources of exposure. Delayed recruitment of some interior participants may limit our ability to examine differential effects of exposure across gestation for this subcohort.

\section{FUTURE PLANS}

Culturally tailored fish consumption advisories are in development to reduce $\mathrm{Hg}$ exposure. An expanded assessment of potentially neuroprotective fish nutrients is under way. A Pesticide Literacy Scale will assist in countering pesticide exposure. Repeated TL measurements of children as an indicator of exposure to environmental toxins will be taken. Neurodevelopmental assessments will take place at 36 and 48 months. An integrated, sharable data management system is in development.

\section{Author affiliations}

${ }^{1}$ Faculty of Medical Sciences, Pediatrics, Anton de Kom University of Suriname, Paramaribo, Suriname

${ }^{2}$ Scientific Research Center Suriname, Academic Hospital Paramaribo, Paramaribo, Suriname

${ }^{3}$ Department of Environmental Health Sciences, Tulane University School of Public Health and Tropical Medicine, New Orleans, LA, United States of America

${ }^{4}$ Foundation for Perinatal Interventions and Research in Suriname (Perisur), Paramaribo, Suriname

${ }^{5}$ Department of Clinical Chemistry Medical Microbiology, Academic Medical Center, Amsterdam, The Netherlands
${ }^{6}$ Institute for Neotropical Wildlife \& Environmental Studies, Paramaribo, Suriname ${ }^{7}$ National Zoological Collection of Suriname/Center for Environmental Research, Anton de Kom University of Suriname, Paramaribo, Suriname

${ }^{8}$ Department of Clinical Chemistry, Academic Hospital Paramaribo, Paramaribo, Suriname

${ }^{9}$ Medical Mission Primary Health Care Suriname, Paramaribo, Suriname

${ }^{10}$ Regional Health Service, Nickerie, Suriname

${ }^{11}$ Department of Public Health, Ministry of Health, Paramaribo, Suriname

${ }^{12}$ Department of Health Policy, Vanderbilt University Medical Center, Nashville, TN, United States of America

${ }^{13}$ Epidemiology, Tulane University School of Public Health and Tropical Medicine, New Orleans, Louisiana, USA

${ }^{14}$ Department of Psychiatry and Behavioral Sciences, Tulane University, New Orleans, Louisiana, USA

Acknowledgements We would like to thank our recruiters Jeetendra Jitan, Nishaira Doerga, Gimradj Dwarka, Sue-Ann Sitaram, Devika Kalika, the Community Health Workers from Nickerie Lilian Singotiko, Henna Mawo, Karien Jodiwongso, Karishma Bhondoe, Jay Kalicharan, Bhairam Goberdan, Azeez Khodabaks, the midwives and laboratory personnel of the Academic Hospital Paramaribo, Diakonessenhuis Hospital, St. Vincentius Hospital, 's Lands Hospital, Mungra Medical Centre, Regional Health Service clinics and Medical Mission Primary Health Care Suriname, the National Zoological Collection of Suriname/Center for Environmental Research staff, Sila Kisoensingh MSc, Dr Richenel Ellecom and Dr Melissa Middleton for their help during the paediatric neurodevelopment assessments and the members of the External Advisory Board and Community Advisory Board for their guidance and valuable advice.

Collaborators We welcome collaboration with fellow researchers working on similar projects. Specific research proposals can be sent directly to the CCREOH Principal Investigators Drs Wilco Zijlmans (wilco.zijlmans@uvs.edu) and Maureen Lichtveld (mlichtve@tulane.edu). Areas of collaboration include further environmental and biomarkers assessment of arsenic exposure, occupational health risk assessments associated with mercury and pesticide exposure, and exposomic analyses including metabolomic assessments.

Contributors ML, JW, HC, EH, AS, SSD and WZ designed and established the cohort. SM-0, AH-M, EH, FAW and AWG participated in the design of the questionnaires. WZ, SM-O, EB, WH, GB, RR and ML were responsible for the continued management of the cohort. GB, RR, AWG and FAW were actively involved with recruitment of participants and collection of non-biospecimen data and biospecimens. JC, JR, PO and GL store, archive and analyze the biomarker samples. AH-M, AS, SM-0, AWG, ML and WZ were responsible for data management. SSD, AWG, RR and EB managed neurodevelopmental testing of babies. JW, LFS, CA and SSD interpreted all results. WZ, JW, HC, AH-M and ML drafted and edited the manuscript. All authors critically reviewed and approved the final manuscript.

Funding Research reported in this publication was supported by the Fogarty International Center of the National Institutes of Health (NIH) under Award Numbers: U01TW010087 and U2RTW010104. The content is solely the responsibility of the authors and does not necessarily represent the official views of the NIH.

Competing interests None declared.

Patient consent for publication Not required.

Ethics approval This study was Institutional Review Board (IRB) approved by both the Government of Suriname and the Tulane University, New Orleans, Louisiana, USA, to consent 1200 pregnant women and their singleton birth children.

Provenance and peer review Not commissioned; externally peer reviewed.

Data availability statement Data are available upon reasonable request. Our cohort study data will be made publicly available upon publication of the results in scientific articles. Questionnaire, registry and biospecimen data could be made accessible in deidentified form after an application process that includes submission of a research plan. We are currently collaborating with Research Triangle Institute International, a global data management enterprise, to develop an integrated database of biospecimen and non-biospecimen data. Once that is fully developed, data can be made available based on a reasonable request to the Principal Investigators. Such requests will be discussed with the full investigator Committee, the Data Management Committee and the Administrative Oversight Committee.

Open access This is an open access article distributed in accordance with the Creative Commons Attribution Non Commercial (CC BY-NC 4.0) license, which 
permits others to distribute, remix, adapt, build upon this work non-commercially, and license their derivative works on different terms, provided the original work is properly cited, appropriate credit is given, any changes made indicated, and the use is non-commercial. See: http://creativecommons.org/licenses/by-nc/4.0/.

\section{ORCID iD}

Wilco Zijlmans http://orcid.org/0000-0002-4080-3858

\section{REFERENCES}

1 Lichtveld MY, Hawkins WB, Ouboter PE, et al. A one health approach to interdict environmental health threats in Suriname. Ann Glob Health 2016;82:444.

2 Silbergeld EK, Patrick TE. Environmental exposures, toxicologic mechanisms, and adverse pregnancy outcomes. Am J Obstet Gynecol 2005;192:S11-21.

3 Ferguson KK, O'Neill MS, Meeker JD. Environmental contaminant exposures and preterm birth: a comprehensive review. J Toxicol Environ Health B Crit Rev 2013;16:69-113.

4 Wigle DT, Arbuckle TE, Turner MC, et al. Epidemiologic evidence of relationships between reproductive and child health outcomes and environmental chemical contaminants. J Toxicol Environ Health B Crit Rev 2008;11:373-517.

5 Lewis M, Worobey J, Ramsay DS, et al. Prenatal exposure to heavy metals: effect on childhood cognitive skills and health status. Pediatrics 1992;89:1010-5.

6 Burns CJ, Mclntosh LJ, Mink PJ, et al. Pesticide exposure and neurodevelopmental outcomes: review of the epidemiologic and animal studies. J Toxicol Environ Health B Crit Rev 2013;16:127-283.

7 Eskenazi B, Kogut K, Huen K, et al. Organophosphate pesticide exposure, PON1, and neurodevelopment in school-age children from the CHAMACOS study. Environ Res 2014;134:149-57.

8 Furlong MA, Engel SM, Barr DB, et al. Prenatal exposure to organophosphate pesticides and reciprocal social behavior in childhood. Environ Int 2014;70:125-31.

9 Quirós-Alcalá L, Alkon AD, Boyce WT, et al. Maternal prenatal and child organophosphate pesticide exposures and children's autonomic function. Neurotoxicology 2011;32:646-55.

10 Rosas LG, Eskenazi B. Pesticides and child neurodevelopment. Curr Opin Pediatr 2008;20:191-7.

11 Bellés M, Albina ML, Sánchez DJ, et al. Interactions in developmental toxicology: effects of concurrent exposure to lead, organic mercury, and arsenic in pregnant mice. Arch Environ Contam Toxicol 2002;42:93-8.

12 Callahan MA, Sexton K. If cumulative risk assessment is the answer what is the question?. Environ Health Perspect 2007;115:799-806.

13 Golding J, Rai D, Gregory S, et al. Prenatal mercury exposure and features of autism: a prospective population study. Mol Autism 2018;9:30.

14 Valent F, Mariuz M, Bin M, et al. Associations of prenatal mercury exposure from maternal fish consumption and polyunsaturated fatty acids with child neurodevelopment: a prospective cohort study in Italy. J Epidemiol 2013;23:360-70.

15 Sapbamrer R, Hongsibsong S. Effects of prenatal and postnatal exposure to organophosphate pesticides on child neurodevelopment in different age groups: a systematic review. Environ Sci Pollut Res Int 2019;26:18267-90.

16 Wang J, Wu W, Li H, et al. Relation of prenatal low-level mercury exposure with early child neurobehavioral development and exploration of the effects of sex and DHA on it. Environ Int 2019;126:14-23.

17 General Bureau of Statistic Studies in Suriname (ABS). Results eighth population and housing census in Suriname (volume 1) demographic and social characteristics and migration, 2013.
18 Ouboter PE, Landburg G, Satnarain GU, et al. Mercury levels in women and children from interior villages in Suriname, South America. Int J Environ Res Public Health 2018;15:1007.

19 Harris PA, Taylor R, Minor BL, et al. The REDCap Consortium: building an international community of software platform partners. J Biomed Inform 2019;95:103208-10.

20 Drury SS, Mabile E, Brett ZH, et al. The association of telomere length with family violence and disruption. Pediatrics 2014;134:e128-37.

21 Cawthon RM. Telomere length measurement by a novel monochrome multiplex quantitative PCR method. Nucleic Acids Res 2009;37:e21.

22 Jayatilaka NK, Restrepo P, Williams L, et al. Quantification of three chlorinated dialkyl phosphates, diphenyl phosphate, 2,3,4,5-tetrabromobenzoic acid, and four other organophosphates in human urine by solid phase extraction-high performance liquid chromatography-tandem mass spectrometry. Anal Bioanal Chem 2017;409:1323-32.

23 Davis MD, Wade EL, Restrepo PR, et al. Semi-automated solid phase extraction method for the mass spectrometric quantification of 12 specific metabolites of organophosphorus pesticides, synthetic pyrethroids, and select herbicides in human urine. J Chromatogr B Analyt Technol Biomed Life Sci 2013;929:18-26.

24 Ouboter PE, Landburg GA, Quik JHM, et al. Mercury levels in pristine and gold mining impacted aquatic ecosystems of Suriname, South America. Ambio 2012;41:873-82.

25 Dellinger MJ, Olson JT, Holub BJ, et al. Mercury, polychlorinated biphenyls, selenium, and fatty acids in tribal fish Harvests of the upper great lakes. Risk Anal 2018;38:2029-40.

26 Ginsberg GL, Toal BF, McCann PJ. Updated risk/benefit analysis of fish consumption effects on neurodevelopment: implications for setting advisories. Human Ecolog Assess 2015;21:1810-39.

27 Clarkson TW, Strain JJ. Nutritional factors may modify the toxic action of methyl mercury in fish-eating populations. J Nutr 2003;133:1539S-43.

28 Davidson PW, Strain JJ, Myers GJ, et al. Neurodevelopmental effects of maternal nutritional status and exposure to methylmercury from eating fish during pregnancy. Neurotoxicology 2008;29:767-75.

29 Strain JJ, Yeates AJ, van Wijngaarden E, et al. Prenatal exposure to methyl mercury from fish consumption and polyunsaturated fatty acids: associations with child development at 20 Mo of age in an observational study in the Republic of Seychelles. Am J Clin Nutr 2015;101:530-7.

30 Taylor CM, Golding J, Emond AM. Blood mercury levels and fish consumption in pregnancy: risks and benefits for birth outcomes in a prospective observational birth cohort. Int $J$ Hyg Environ Health 2016;219:513-20.

31 Golding J, Gregory S, Emond A, et al. Prenatal mercury exposure and offspring behaviour in childhood and adolescence. Neurotoxicology 2016;57:87-94.

32 USEPA. Mercury study report to congress volume $V$ : health effects of mercury and mercury compounds. Washington, DC: United States Environmental Protection Agency, 1997: Vol 5. 1-348.

33 Zilversmit L, Wickliffe J, Shankar A, et al. Correlations of biomarkers and self-reported seafood consumption among pregnant and nonpregnant women in southeastern Louisiana after the Gulf oil spill: the GROWH study. Int J Environ Res Public Health 2017;14:784.

34 Castaño A, Cutanda F, Esteban M, et al. Fish consumption patterns and hair mercury levels in children and their mothers in $17 \mathrm{EU}$ countries. Environ Res 2015;141:58-68.

35 Abdoel Wahid F, Wickliffe J, Wilson M, et al. Presence of pesticide residues on produce cultivated in Suriname. Environ Monit Assess 2017;189:303.

36 Abdoel Wahid F, Hindori-Mohangoo A, Wickliffe J, et al. Dietary exposure to pesticides in Tannia in pregnant Surinamese women. ISEE Conf Abst 2018;2018. 